\title{
Variations
}

Variations

Revue internationale de théorie critique

$12 \mid 2008$

Wärmestrom

\section{Au bord du Kiaï}

Subjectivités rebelles, démocratie sauvage, espace public oppositionnel

\section{Lucia Sagradini}

\section{(2) OpenEdition}

Journals

\section{Édition électronique}

URL : http://journals.openedition.org/variations/239

DOI : $10.4000 /$ variations.239

ISSN : 1968-3960

\section{Éditeur}

Les amis de Variations

\section{Édition imprimée}

Date de publication : 21 décembre 2008

\section{Référence électronique}

Lucia Sagradini, « Au bord du Kiaï », Variations [En ligne], 12 | 2008, mis en ligne le 01 janvier 2012 consulté le 20 avril 2019. URL : http://journals.openedition.org/variations/239 ; DOI : 10.4000/ variations.239

Ce document a été généré automatiquement le 20 avril 2019

Les ami•e•s de Variations 


\title{
Au bord du Kiaï
}

Subjectivités rebelles, démocratie sauvage, espace public oppositionnel

\author{
Lucia Sagradini
}

\section{NOTE DE L'ÉDITEUR}

Première publication sur www.theoriecritique.com, « Wärmestrom, le courant chaud en sciences humaines ", Hiver 2008/2009, pp. 22-46

1 Qu'est-ce que penser? Pour chacun d'entre nous, la question va entraîner une réponse singulière, pétrie de notre expérience, mais elle peut également varier en fonction du moment même où cette question se pose.

2 Entre 2005 et 2006, la société française connaît un certain nombre d'événements que l'on pourrait qualifier de nouveaux. L'un d'eux est ce que les médias ont qualifié de « crise des banlieues ». La société dans son ensemble est traversée par cette " émeute » qui parcoure le territoire, et qui explose dans des endroits éloignés les uns des autres : les jeunes des quartiers populaires se révoltent.

3 Le monde entier vient alors regarder cet événement, il s'ensuit un flot d'images assez homogènes, montrant des jeunes jetant des pierres, des forces de l'ordre chargeant dans un climat apocalyptique, ainsi que de vaillants journalistes, tenant courageusement leur micro, devant des voitures incendiées d'où les flammes s'échappent encore.

4 Je suis alors en train de réaliser une thèse sur la dictature en Argentine, et plus particulièrement je travaille à reconstruire la tension paradoxale entre l'incidence de ce régime autoritaire sur les pratiques culturelles et la subjectivité, que j'appelle «le trauma », et de l'autre les formes de résistance et d'élaboration qui y voient le jour.

5 Ainsi, ai-je débuté une pensée de la démocratie et des formes de résistances à l'oppression par une reconstruction du panorama argentin de la culture et des pratiques artistiques entre 1976 et 1995. 
6 D'une certaine manière, ce travail de réflexion sur la démocratie et l'oppression, dirigé dans une thèse particulière, ce travail s'est également nourri du moment auquel cette pensée se déployait, habitée par cette «crise des banlieues». Désormais, cet opus universitaire était irréductiblement lié à l'observation de ce phénomène autre, venu des banlieues françaises, et qui entrait en résonance.

7 Aujourd'hui, la thèse achevée, c'est cet alliage étrange entre une réflexion sur l'Argentine, et une autre sur la « crise des banlieues » que je souhaite interroger, forte du sentiment que la question de la démocratie peut se parcourir de l'une à l'autre, tout en y associant un troisième élément : ce qui s'est déroulé à Montréal Nord depuis août 2008.

8 Ces trois phénomènes singuliers offrent ainsi la possibilité d'ouvrir une réflexion sur les situations de débordements et de révoltes. Ils sont trois exemples empiriques marqués par leurs statuts de situation qui échappe au contrôle, de rejet de l'État et de débordement populaire.

9 En parallèle, pour les analyser et pour poursuivre une réflexion sur les sociétés démocratiques contemporaines, j'ai saisi un instrument vital : l'ouvrage d'Oskar Negt, L'espace public oppositionnel' ${ }^{1}$. Un livre qui allait être un choc dans l'univers théorique dont j'avais hérité.

10 Car, il existe chez Oskar Negt cette capacité de penser double, venue tant de la philosophie, que de la sociologie, en cela il s'inscrit dans le droit-fil d'Adorno, car il y a cet intérêt pour le monde tel qu'il est, et dans ces manifestations.

«L'effort pour définir cet espace public oppositionnel et vivant constitue pour moi une interrogation centrale de recherche, à la différence de Jürgen Habermas qui parle d'une simple «variation plébéienne» de l'espace public bourgeois, dans son ouvrage du même nom...» (Negt, p.158).

11 Ainsi, chez Negt, il se présente, tout à la fois, ce projet de réaliser une critique de la domination, qui hérite de l'Ecole de Francfort, et cette capacité de repenser au-delà du marxisme les formes du social. Ce faisant, Negt concrétise l'existence d'un espace public oppositionnel qui est une forme hétérogène qui est ainsi redéfinie, re-formalisée par l'auteur en s'appuyant sur ce courant chaud qu'est la théorie critique ${ }^{2}$. Et dans cette reformalisation du social, il y a la possibilité d'articuler les agir-s des individus et de sortir d'interprétations clivées qui nient la complexité de la société et de la catégorie des "petits", les exclus, les précarisés, ainsi que les classes moyennes toujours potentiellement « marginalisable » par une société axée sur le capitalisme qui détruit ce sur quoi elle repose.

\section{Trois cas empiriques et un concept}

12 Ainsi, il s'agit ici d'initier une réflexion et une analyse de ces situations hétérogènes de débordement que sont les manifestations en Argentine, la « crise des banlieues » et les « émeutes » de Montréal-Nord.

Dans ce dessein, il faut d'abord souligner que ces trois phénomènes portent leur propre singularité, mais qu'ils offrent également par l'usage du concept d'espace public oppositionnel une mise en commun d'expériences venues des couches populaires de la société. Cet outillage conceptuel va ainsi nous permettre de re-parcourir les événements en dégageant une pensée de la politique.

14 Les trois cas sont aussi marqués par une différence aussi de contenus. 
15 En ce qui concerne l'Argentine la réflexion tourne autour des manifestations de rue qui eurent lieu en Argentine à partir du krach économique de 2001, mais sont également relié à des expériences antérieures de pratiques démocratiques de rue. Un des points essentiels pour nous étant de souligner le travail de l'inventivité qui a parcouru les révoltes de 2001 tout en les reliant à la pratique de l'escrache initiée en 1995, et à celle du siluetazo qui eut lieu en 1983.

\section{Une expressivité démocratique en dehors de l'Etat}

De mon précédent travail, j'ai gardé le goût d'interroger les formes esthétiques qui se jouent dans le cadre d'expérience politique. Ainsi, je me suis intéressée à la pratique du siluetazo en 1983, qui concentrait un projet artistique performatif et une pratique politique de lutte et de dénonciation de l'État argentin, soutenu et accompagné par les Mères et Grands-Mères de la Plaza de Mayo, puis par l'ensemble des citoyens. De là, je suis arrivée aux escraches et aux formes d'art callejero qui ont accompagné les manifestations, et notamment celles de 2001 lorsqu'il y a un rejet tant de la gouvernance et des formes traditionnelles de la politique que de la situation économique avec un krach économique.

17 L'inventivité au service de la politique qui se manifeste en Argentine, et son soutien à l'expression d'un projet d'une société autre, redouble les consonances politiques des manifestations et de pratiques d'invention. Comme l'occupation et l'autogestion des usines, le déploiement des erroristes, et l'inventivité pour faire face à la crise avec l'usage du troc, d'une monnaie propre au sein de la capitale ou de la décision des artistes et des gens de la culture de jouer gratuitement.

18 L'expérience d'un autre type de vie et l'expression de projet de vie ensemble autre, de souhaits et d'intérêt allant bien au-delà de l'inquiétude en terme seulement financier. Elle est le signe d'une grande vitalité des couches populaires, de leur rassemblement manifeste à des moments clés, de leur désir de projet social qui déborde la question de la somme d'argent que l'on peut gagner ou non. Cette forme inventive, qui accompagne la lutte et les révoltes de 2001, est ainsi émettrice d'un projet de société et d'une quête de dépassement qui se constitue dans cet espace public oppositionnel.

Lorsque le krach économique de 2001 a entraîné l'écroulement de l'ordre économique et l'appauvrissement généralisé des citoyens, allant jusqu'au désespoir, nombre d'individus se sont emparés de la situation pour inventer de nouvelles formes de luttes d'opposition et de rejet de cet état des choses. Caceroleros et piqueteros ont pris la rue, mais il s'agissait aussi d'aller au-delà, en faisant surgir d'autres manières de vivre et de s'approprier sa propre vie. Dans cette situation violente, les êtres se sont démarqués par leur capacité d'agir, qui a débordé toutes les conditions imposées du dehors. Toutes ces manifestations sont autant d'expressions de formes se donnant comme alternatives à l'État et l'organisation sociale qu'il impose.

Alors que les sociétés contemporaines sont tournées vers une expropriation,

"Il ne s'agit plus de l'expropriation des êtres humains, de leur force de travail, mais d'une dépossession supplémentaire de leur imagination, de leurs possibilités existentielles et de leur bonheur ...» (Negt, p.43).

Les pratiques d'invention qui traversent l'espace public oppositionnel argentin indique cette lutte contre cette dépossession. 
21 Le travail de l'imagination (Negt, pp.79-85) dont parle Negt, en reprenant le terme à Freud, est bien là, il indique la source à laquelle la résistance et le débordement des couches populaires peuvent se soutenir pour contrer et contrecarrer les projets de société mis en place dans la sphère politique et financière (Negt, PP.40-54). Le gouvernement de Kirchner n'a pu ainsi empêcher les usines autogérées de tourner, il a aussi été poussé à reprendre une politique de justice visant les responsables de la dernière dictature. Il y a ainsi des temps et des moments qui doivent être perçu comme des brèches qui mettent à mal le pouvoir, et qui le contraignent à résister.

Cette inventivité à l'œuvre dans les manifestations de rue semble aussi être le signe d'un espace public oppositionnel qui peut et se mobilise lorsque cela devient un impératif, qui n'est pas pris dans l'atonie et qui peut ouvrir sur des changements décisionnels. Il y a alors bien une incidence de cet espace public oppositionnel sur la sphère du politique, malgré le fait que celle-ci comme la souligné Victor Harmony, est totalement réifié dans une expression péroniste archaïque.

Il faut aussi dire que c'est aussi de cet espace public oppositionnel que sont venus les changements dans le monde politique, la succession des présidents en un temps record, indique bien que si cet espace public oppositionnel se manifeste, alors de grands changements peuvent se produire. Il est nécessaire d'insister sur le fait que cet espace public oppositionnel, marquée par la différence de ses pratiques politiques et de son extériorité avec le champ traditionnel de la politique, a pourtant une réelle incidence sur le champ politique traditionnel et les institutions.

Ce premier exemple est ainsi tourné et engagé dans une réflexion soutenue depuis la thèse et qui tient à souligner la capacité d'invention des couches populaires dans une et des périodes de tensions politiques.

Le second est un peu - inversement au travail de thèse - au cœur de ce nouveau dispositif qu'est cette recherche pour la Chaire Mondialisation, Citoyenneté, Démocratie. Il concerne les « émeutes des banlieues » en France en 2005. Il est tout à la fois le moteur de ce travail qui s'initie avec ce texte, et aussi moins chargé de contenu puisqu'il débute.

Ce second phénomène se caractérise par le soulèvement de la jeunesse populaire, venue des quartiers populaires et de la périphérie, à la suite de la mort de deux jeunes. Cette mort est due à l'électrocution des deux jeunes garçons qui, pour échapper à l'interpellation des policiers, se sont réfugiés dans le bâtiment d'un transformateur électrique, et où la police les a laissés, sachant sciemment le danger qu'ils courraient. Ces jeunes étaient mineurs, ils ne faisaient rien de répréhensible, mais ils ont préféré s'enfuir devant les forces de l'ordre plutôt que de subir leur contrôle ${ }^{3}$. Leur mort a entraîné des révoltes durant plusieurs jours et dans toute la France, des altercations avec la police ont eu lieu durant ces journées, ainsi que des saccages et des dégradations de biens communs. La réponse répressive de l'État français n'a pas tardé. Un couvre-feu a été établi, le dernier datant de la guerre d'Algérie, et il s'accompagne de l'interpellation et des comparutions extraordinaires de quantité de mineurs sans casiers judiciaires ${ }^{4}$.

Les médias, quant à eux, ont été totalement happés par cet événement où la jeunesse populaire et les origines étrangères -d'Afrique ou du Maghreb- étaient systématiquement soulignées stigmatisant les jeunes et entraînant la compréhension de cette jeunesse comme un « danger pour la société ». 
$$
\text { Pierre Clastres, car tous deux travaillaient de manière atypique la question de la politique }
$$
en la déplaçant de son champ convenu de l'Etat et des institutions ${ }^{6}$.

\section{L'Espace public oppositionnel}

\section{Le décalage de la politique et de l'Etat}

Comment compter rassembler ces événements et pour quelle pertinence? Telle pourrait être la question première. Il a déjà été dit que c'est l'usage du concept de Negt qui ouvrait la piste de cette réflexion. Ainsi, le premier point commun qui me conduit à considérer ces trois cas singuliers de manifestation populaire de politique est lié au chapitre de Negt sur l'Allemagne année zéro (Negt, pp. 143-153).

En effet, les pratiques de manifestations artistiques et politiques en Argentine concentrent deux points essentiels touchant aux formes de révolte. La première est que face à l'iniquité de l'État -et ici dans sa forme autoritaire, dictatoriale- les individus, du moins certains, sont travaillés par le désir de se révolter, de résister à l'iniquité de l'État. Les formes de cette résistance rencontrées dans la thèse m'indiquaient qu'elles pouvaient être extrêmement variées. Que chacun trouvait, seul ou-et dans des collectifs, des pratiques de résistance, allant du port de la barbe à la manifestation collective, et à la performance artistico-politique.

Le second point est que ce travail de thèse me sensibilisait sur les moyens de faire de la politique -chaque acteur du social ayant sa perception de la manière d'entrer en révolte contre l'État et le recours à l'invention et à l'imagination dans les manifestations de rue avaient éveillé mon attention.

Ce second point faisait entrer également en résonance le texte de Negt et la lecture de en la deplaçant de son champ convenu de letat et des institutions.

Variations, 12 | 2012 

politique, une brèche dans laquelle j'allais m'engouffrer puisque dans le passage Allemagne année zéro, il y avait une réponse concluante pour mon travail (Negt pp.143-153).

Par exemple, le fait de constater que les cadres et les différents relais qui formaient le monde administré et faisaient vivre les institutions, étaient pour la plupart les mêmes avant et après la dictature trouvait sa correspondance dans l'extrait de Negt. En effet, l'auteur indique bien que la démocratie n'est pas dans l'État au moment de la libération du nazisme, mais dans les pratiques des individus (Negt, pp. 145-146). Cet aspect de la démocratie qui se décale de l'existence des institutions, que souligne Negt, allait non seulement me servir durant la thèse tournée vers l'analyse de ces pratiques artistiques et politiques chargée d'un potentiel subversif, et aussi dans l'analyse de leur participation à la construction démocratique en Argentine.

Ce décalage touchant aux formes de la démocratie que Negt installe dans l'ouvrage est ainsi le levier utilisé pour considérer "la crise des banlieues» en France et les «bouleversements" de Montréal-Nord. Car, là encore, il s'agit de considérer non seulement les "émeutes » différemment, mais de les faire passer dans la catégorie de "révoltes» urbaines afin d'enrichir l'analyse de ces phénomènes comme étant l'expression d'une révolte contre l'État.

En effet, le point d'origine de ces révoltes me semble d'une importance capitale, car il s'agit de soulèvement contre l'iniquité de l'État. Les révoltes urbaines sont bien des expressions populaires de la politique, car elles signalent en premier lieu un refus d'un ordre des choses reconnu par les jeunes, et qui est caractérisé par l'abus de l'État et aussi l'attitude du gouvernement de soutenir les forces de l'ordre alors même qu'elles ont eu une action illégitime d'usage de la violence.

D’ailleurs, cette réaction de la jeunesse a trouvé également la solidarité des aînés, ou du moins une compréhension alors même que la violence embrasait les jeunes.

Une attitude que l'ensemble de la société et de l'opinion publique n'a pas partagé, car le non-respect et les dégradations étaient les lieux de la focalisation médiatique bien avant la question de condamner les abus d'autorité de l'État -un couvre-feu comme en temps de guerre n'a pour ainsi dire pas choquer, ni même le fait que des mineurs innocents soient morts victime de leur peur devant la police.

\section{La conflictualité de la politique et la question des subjectivités}

Ici, se tient la force vitale de Negt, car loin d'écarter ce type d'événements de l'analyse de la politique, son concept d'espace public oppositionnel permet de les inscrire à l'intérieur. En effet, Negt a cette originalité de réinscrire dans un face-à-face tendu théoriquement avec Habermas le caractère conflictuel de la démocratie et de l'espace public.

44 À la vision idéalisée et «bourgeoise » d'Habermas d'un espace public lieu d'échange de parole et déploiement de la représentativité démocratique, Negt a le goût de construire à son revers un espace public oppositionnel - un espace populaire de la politique où des révoltes urbaines et les manifestations de rue que nous avons vu plus avant trouve leur place. Celle de rendre compte de l'activité et des formes particulières de démocratie populaire. Il n'y a pas que l'espace de la représentativité pour être espace de la politique, et grâce à Negt, la subjectivité et les expériences des couches populaires de la population 
connaissent une visibilité. C'est-à-dire qu'elles peuvent enfin être saisies dans leurs portées théoriques.

Les révoltes urbaines conduisent aussi à la mise en relation avec un autre aspect de cette conceptualisation de Negt. Car, le modèle d'espace public habermassien qui est comme le reflet d'une société idéalisée où la représentativité et ses formes -les Clubs, la Presse, les Associations, l'Assemblée- seraient l'expression de la politique et la forme de la démocratie est alors totalement caduque pour considérer et comprendre les révoltes urbaines.

Alors que Negt apporte la contradiction dans l'espace public, il réintroduit les couches populaires - dixit le prolétariat - dans l'expression de la politique. Et, dans le même temps, il apporte aussi la contradiction à la définition habermassienne d'un espace public, qu'il interprète comme espace public «bourgeois", auquel, par la participation des médias, cet espace devient un "théâtre des illusions ", et surtout un espace allant vers l'homogène et du coup écartant le conflit.

En réalité, Negt nous montre comment cet espace public dans la définition habermassienne est celui qui manifeste la loi du plus fort, tout en se séparent et en niant les expressions venues des couches populaires.

"À l'intérieur de cet espace public, le fait de "posséder de l'expérience" consiste à disposer de savoir des dominants, un savoir spécialisé au vu de l'utilisation appropriée de cet espace public. » (Negt, p. 65)

Dans cette situation, la perception de l'événement - ici, des révoltes urbaines - ne répond qu'aux représentations des dominants. Il s'agit donc d'un espace inique, de, par sa construction, qui isole les couches populaires, et qui les isole également de leurs propres expériences (Negt, pp.57-62).

La force d'Oskar Negt est que, contrairement à Habermas, il nous propose de saisir la lutte politique qui s'exprime dans les couches populaires. Il nous propose de voir les choses différemment, et notamment, les événements en sortant d'une vision mutilée par une représentation du social qui nie l'expérience politique et populaire. Grâce à lui, il est désormais possible de réinterpréter différemment l'espace public et les phénomènes, car il introduit dans sa vision de la société la conflictualité, et le rapport mutilant à l'expérience venu de l'espace public traditionnel.

« Nous ne devons pas nous borner à faire participer les masses aux décisions politiques, nous contenter de les informer, et de les pousser à s'engager; il s'agit plutôt de voir que l'enjeu central de la libération de soi nécessite un "espace public prolétarien" autonome, susceptible de porter cette libération... » (Negt, p.38).

Il fait alors surgir la notion de l'espace publique oppositionnel et ainsi il réintroduit dans le champ de l'analyse le caractère conflictuel du social. Qui se trouve du côté de la démocratie. Ce faisant, il ne s'agit pas pour lui de gommer le premier des deux espaces, mais plutôt de lui apporter un acolyte (Negt, p.67).

51 Ce point est particulièrement intéressant et complexe chez Negt, car il ne s'agit pas de faire table rase de l'espace public bourgeois. De fait, cet espace public traditionnel est aussi à son origine dans une quête révolutionnaire, et une quête de liberté. Il insiste d'ailleurs sur la nécessité que les couches plébéiennes saisissent et défendent cet espace public, alors même qu'il leur est désormais en grande partie défavorable et aliénant, puisque cette partie de la population n'a pas de place réelle en son sein, (voir Negt, p. 60 et suivantes). Nous sommes à cet endroit dans une dialectique entre espace public bourgeois et espace public oppositionnel. 
52 Negt souligne comment cet espace public bourgeois ne sert pas aux petits, car l'expérience réalisée en son sein appartient au domaine du privé, (Negt, p. 60). Donc, il insiste sur le fait que cet espace public bourgeois ne permet pas d'organiser et de tirer parti des expériences des couches populaires, ou de fédérer leur intérêt. Cet espace public bourgeois est de fait occupé par l'État et non par le rebelle. De fait, il y existe donc une exclusion des couches sociales qui ne peuvent saisir ce mode bourgeois d'expression et de politique, et qui n'ont pas non plus les moyens d'y participer. Il est donc un espace qui ne peut pas accueillir ou recueillir les expériences venues des sphères oppositionnelles. Car « il faut accepter le fait que l'État occupe l'espace public, et non pas le rebelle... », (Negt, p.63). Raison pour laquelle les révoltes urbaines sont ainsi si mal comprises par l'espace public traditionnel.

53 L'espace public oppositionnel est en effet une partie qui vient compléter l'espace public traditionnel. Negt nous permet ainsi de saisir que ce qui se joue dans et par l'espace public bourgeois n'est pas la totalité ni de la politique, ni de la démocratie, il fait surgir dans le champ théorique un élément complémentaire qui participe à la politique tout en étant constamment nié par le premier espace. Cette négation entraîne ainsi et participe à l'occultation des expériences et à l'isolement des individus qui dès lors ne peuvent pas par le biais de l'espace public traditionnel saisir et comprendre leur propre expérience (Negt, p.68). Cette action d'isolement et d'appauvrissement de l'expérience par l'espace public traditionnel est un des moyens de maintenir un ordre établi tourné sur la productivité et écarte l'authenticité du domaine de l'expérience humaine.

54 Penser avec Negt et son concept c'est également le seul moyen de réintroduire le dépassement dans la compréhension des situations de troubles, et les expériences de débordements démocratiques. En effet, il est impossible alors de ne pas saisir les phénomènes de la rue comme autant de manifestation de l'aspiration à saisir sa vie, à comprendre sa propre expérience et surtout lorsqu'il y a une forte mobilisation spontanée contre les forces de l'ordre suite à un abus -cela vaut pour les trois cas- ces révoltes expriment ce désir d'équité et de justice, et une aspiration à une autre vie, à autre chose.

Negt s'attache donc à penser la critique de la domination en créant ce cadre conceptuel de l'espace public prolétarien, mais il le pense dans le souci de la libération de soi, et en cherchant à soulever que cet espace public oppositionnel est l'espace au sein duquel les individus arrivent à donner expression à leurs aspirations essentielles et à chercher leur libération. La place nodale faite à la subjectivité et à l'intérêt porté à l'individu en tant que personne, elle est comme le moteur à partir duquel va se dérouler l'action. L'espace public oppositionnel va être le terrain qui va permettre cette quête individuelle en l'arrimant à un espace collectif. (Negt, p. 38).

56 L'intérêt pour l'espace public oppositionnel est ainsi dû au fait que ce concept permet de faire émerger des aspirations autres, ainsi que d'autres modalités, formes de vie, d'actions, de pratiques, différentes de celles de la bourgeoisie existent. Cet espace public oppositionnel est en fait comme le réservoir, le lieu d'expression de l'altérité par excellence. Comme le signale Negt, il s'agit pour lui de créer et d'opposer_à la légitimation démocratique de la domination, un concept offensif et positif qui puisse lutter contre ce principe de légitimation, (Negt, p. 33). 
Et particularité qui m'intéresse au plus au point un concept qui parle non seulement de la raison, mais aussi et surtout des affects, leur image de la libération, et leurs intérêts immédiats.

\section{Médias et double réalité}

En France, la lecture faite par les médias est axée sur cette impossible reconnaissance du conflit politique qui se joue dans ces révoltes urbaines. La presse, dans son ensemble, a, d'ailleurs, refusé de parler de révoltes urbaines, elle a préféré désigner les événements par le terme "d'émeutes ». La première action des médias a été de nier le caractère politique de l'événement. Les intellectuels quant à eux tendent à analyser la situation comme un "en-deçà " politique qu'il faut chercher à saisir. L'action de l'espace public traditionnel à l'encontre de ces jeunes a ainsi été de leur nier une activité politique.

Dans une mesure moins grande, la presse populaire distribuée gratuitement à Montréal, depuis septembre, a eu une action néfaste, elle aussi, car elle entérine la question des gangs. Un certain nombre d'articles sont ainsi diffusés sur le problème des gangs et cette question est immédiatement accolée à l'identification d'une population : les haïtiens. Là, encore, cette interprétation sort les acteurs sociaux d'un rapport et d'une action politique et les met du côté de la délinquance. Dans le même temps, cette orientation de la presse stigmatise alors un peu plus des personnes vivant dans les quartiers populaires.

«il n'y a pas d'un côté les « délinquants» ou « la racaille» et de l'autre les «bonnes victimes ». Tous les habitants de ces quartiers sont victimes: de l'inattention sociale, plus encore du mépris avec lequel on nie leurs droits, leurs valeurs, leurs existences mêmes, et dont le droit de cité n'est compris que comme une quasi-assignation à résidence. C'est pourquoi les révoltes relèvent bien d'une forme de rationalité politique, à laquelle nous ne sommes pas habitués certes, mais dont le mode d'expression enragé manifeste combien il est urgent de baisser le seuil de l'audible en politique pour recevoir les demandes de justice sociale de ceux qui sont exclus de tout ${ }^{7} »$.

60 Ici, il y a cette concentration d'une analyse qui cherche à avoir un autre regard sur les révoltes urbaines de 2005, mais il s'y présente également les limites propres de la pensée inscrite dans le cadre reçu de l'espace public bourgeois. Premièrement, ce n'est pas une articulation suffisante que de penser en termes de victime ce qui s'est produit en 2005. Car, il y a alors une occultation de l'agir de la jeunesse qui se révolte et conteste l'État.

Deuxième point, et il s'agit là encore d'une pensée qui s'inscrit dans les limites données par le cadre traditionnel : ce n'est pas une analyse critique que de dire qu'il faut baisser le niveau de l'audible. Car, en réalité, cette sphère de l'espace public bourgeois, dégagé par Habermas, fait un choix structurel de ne pas entendre, il ne s'agit pas de nous baisser, il s'agit de comprendre qu'il y a un autre type d'espace public, et d'autres formes d'expression et d'expériences, que celles et ceux que l'univers bourgeois désigne comme valides et audibles. Ne sont reconnues que les formes que nous reconnaissons, c'est-àdire, les formes dominantes d'expressions. Il est temps de saisir qu'il y a d'autres formes de l'expérience qui sont le fruit des "petits", des "vaincus ", et qu'il nous faut saisir comme telles sous peine de rater le rendez-vous avec la praxis et l'empirique,_car nous ne pourrons sans cela les saisir.

62 Il nous faut donc conceptualiser ces événements et ces actions grâce au travail de Negt qui permet de les saisir dans leur enjeu politique. 
Le point découlant de cette compréhension de l'action de l'espace public traditionnel est l'enjeu de la visibilité.

En réalité, l'espace public bourgeois, habermassien, est non seulement l'espace qui est le réceptacle de ceux qui ont l'usage de la parole et de la «bonne parole »; mais aussi celui de la visibilité des acteurs. Est reconnu ce qui fait partie et marque son appartenance à cet espace public bourgeois. Le propre des milieux populaires est de ne pas pouvoir apparaître, de ne pas être reconnu dans ces formes mêmes d'existence. Il y a ainsi une souffrance provoquée par l'absence de visibilité Il y a un invisible qui est un insupportable et qui opère dans le déni social de l'action collective et organisée de la société par ces institutions et son État sur des minorités exclues socialement.

D'ailleurs, une piste pertinente serait d'examiner méticuleusement les modes d'apparition, qui sont privilégiés pour rendre compte de l'existence de ces « exclus ». Rapidement, il est possible de voir alors que le cliché, l'usage d'images négatives et stigmatisantes sont diffuses dans les médias. La couverture médiatique -sphère dominante- fait ainsi toujours le choix de marteler ces images, écrites ou visuelles, tournées vers des représentations de violence et d'archaïsme. La peur est en conséquence un moyen de maintenir des clivages sociaux sur lesquels repose la reproduction sociale.

6 Comme nous l'avons vu, les thématiques de la «victime » et de «la misère » sont en contraposto les représentations qui peuvent guider un certain nombre d'analyses théoriques et académiques. Cependant, ces représentations ne peuvent suffire, car elles ne permettent pas d'expliquer l'action - les révoltes urbaines - et à la considérer comme une action politique. Il serait bon de poursuivre ce projet de recherche en analysant les apports venus du champ académique ainsi que leurs limites vis-à-vis du phénomène ${ }^{8}$.

Au contraire, je pense que l'action politique bien que pouvant être lié à un sentiment négatif, en quête de plus de justice ou de reconnaissance sociale, est le fruit de l'aspiration à la liberté, à la démocratie et ce faisant au dépassement comme la déjà admirablement indiquer Françoise Proust ${ }^{9}$. Ces trois phénomènes manifestent chacun à leur manière cette quête démocratique.

8 De plus, Negt signale que le concept de démocratie est lui-même une idée que chaque génération par ses luttes spécifiques va se réapproprier et le nourrir de ce rapport spécifique. Il devient alors indispensable de faire des recherches sur les formes de la lutte, car elles sont aussi porteuses de cet imaginaire de la démocratie renouvelée et entravée.

69 Par exemple, l'apport des «sans papiers " à la vie démocratique est ainsi un angle à étudier, car ils réalisent de leurs constants efforts pour étendre les limites de la société qui les « reçoit ». De plus, la question des révoltes urbaines a cette particularité de ne pas être encore accompagnée de mots ou de construction politique «traditionnelle » et aussi d'avoir lieu en étant en grande partie niée. La question du dépassement n'est pas, et nous le verrons, avec chacune des situations singulières, détaché de la question de la violence qui elle est du côté archaïque de l'action négative ${ }^{10}$. Toutes ces problématiques vont devoir se travailler et je les aborderais plus avant.

Mais pour l'instant revenons à l'expression des révoltes urbaines et des manifestations comme étant l'expression d'une démocratie sauvage. 


\section{Démocratie sauvage et le « contre l'État » de la société}

71 Dans une constellation d'auteurs marginaux, la pensée de Negt permet ainsi d'éclairer différemment ces phénomènes, il fait la part belle au conflit dans la démocratie, il concourt à rendre visible les luttes des couches populaires des sociétés démocratiques contemporaines. Il complexifie la relation attendue à la démocratie, il peut porter en lui la compréhension tant d'une démocratie sauvage que des subjectivités rebelles.

À la lecture de La démocratie contre l'État, écrit par Miguel Abensour, cette notion de démocratie sauvage est profondément fertile ici, car elle développe l'idée qu'il y a deux courants, mieux, deux agir politiques ${ }^{11}$. D'un côté, il y a cet agir qui cherche à transformer le régime politique, la construction du pouvoir et qui va dans la transformation de l'Etat, qui conduit à une conquête et aussi à une réification des formes institutionnalisées de la politique (Abensour, PP.109-110). De l'autre, un second agir, une démocratie sauvage, qui elle va chercher à éroder la forme prise par l'État ${ }^{12}$.

En effet, dans cette situation où les formes de la contestation et de la politique se passent et se déroulent en dehors des cercles convenus et attendus de la politique de partis, et notamment des partis qui traditionnellement ont été ceux de « la classe ouvrière ». Il y a bien une indépendance et une auto-désignation, autoformation par les espaces où se construisent les luttes politiques.

Il y a bien un écart, et il me semble que l'espace public oppositionnel vient palier cette absence de cadre théorique pour comprendre les nouvelles formes d'organisation des luttes politiques. C'est pourquoi il est important de souligner qu'il y a bien une crise de la représentation politique et dans le même temps une invention de la chose politique.

"Ce faisant, ce que Marx donne à comprendre, dans le dialogue qu'il entretient avec Hegel, Moses Hess et Spinoza, c'est une pensée du politique en son autonomie - hors de toute prétention à le dissoudre dans une genèse empirique -; une pensée du rapport insuperposable entre l'État et la démocratie et d'un jeu permanent d'inadéquation; une pensée affirmative de la souveraineté qui n'est pas seulement d'essence démocratique, quelle que soit la forme d'État, mais qui trouve dans la démocratie comme telle son rapport même d'auto-institution continuée » (Abensour, p. 114).

Ainsi, cette démocratie sauvage est en non-coïncidence avec l'État. Les révoltes urbaines et les luttes dans nos trois cas sont le signe aussi manifeste d'une démocratie qui se réalise, au moment-même où la gouvernance prend en charge les formes archaïques qui tirent le plus vers le bas la société. L'espace public oppositionnel est le fruit et la manifestation, l'expression des formes des minorités conflictuelles et oppositionnelles de la société.

Évidemment, c'est ce second type d'agir qui nous intéresse, car il vient entraver la réification de la politique en sa forme étatique, ouvrir des brèches et proposer d'autres formes pour le vivre ensemble, avec cette idée sous-tendue $d$ agir de concert. De laisser échapper le pouvoir dans sa forme coercitive, pour entrer en quête d'une action de concert, où la forme démocratique se noue à la forme égalitaire (Abensour, p.110).

Il y aurait donc dans la révolution et la transformation de l'État sa propre tentation vers une forme absolutiste, où l'État cesse de protéger les individus pour se protéger contre les individus, "parce que l'État représente pour la démocratie un danger permanent de dégénérescence ", (Abensour, p.108). Ce mouvement vers un durcissement qui est contenu dans les formes démocratiques modernes mêmes, est alors entravé par ce second agir, un agir populaire, qui a pour volonté de venir conteste cette édification de la forme étatique 
qui progressivement se coupe de son aspiration et du souffle démocratique qui est à son origine (Abensour, p.108).

Imaginer cette démocratie sauvage, pratiquée par les exclus, les vaincus, et prendrait la forme d'une démocratie sauvage, il serait alors la modalité par excellence de la conquête démocratique et serait lié au fait que la loi les exclut d'autant - frappés d'inexistence par le droit lui-même.

Il est d'ailleurs intéressant de télescoper cette idée de démocratie sauvage avec la réactualisation $\mathrm{du}$ terme sauvage. Car, ce dernier connaît dans le champ de l'anthropologie / ethnologie un réinvestissement, en commençant par Lévi-Strauss, avec La pensée sauvage ${ }^{13}$ et qui peut faire écho ici, à une ré-appropriation après le "sauvageons" de Chevènement. Cette mise en résonance va aussi jusqu'à la forme verbale qui a accompagné l'expression de défiance, de préjugés et de racisme tournée vers les couches populaires et la jeunesse qui sont aussi le lieu où s'expriment l'altérité. À la parole provocatrice et péjorative, de Sarkozy, de "racaille ", certains ont répondu par la phrase du genre « alors j'en suis aussi » ou « fière d être une racaille ${ }^{14}$ ».

La démocratie sauvage, concept éminemment bénéfique dans le travail de mise en brèche et de valorisation de formes d'actions venant entraver la réification des formes du pouvoir, et alors comme rentrant en harmonie avec une réutilisation de l'idée du "sauvage ", celui qui en fait agit pour le bien commun et permet à la liberté d'être toujours exprimée comme une quête d'idéal politique.

\section{Des trois cas de démocratie sauvage}

81 Par le choix de nos trois cas, argentins, français, et canadien-québécois, il s'agit ainsi de considérer ces phénomènes en retraçant leur unité, dans le sens, où ils sont, à chaque fois, l'expression d'une révolte contre l'état de la société, surgie de ses groupes oppositionnels. Ce travail est ainsi une mise en forme et une analyse des subjectivités rebelles, qui peuvent ne pas être, ou peu, organisées, qui médiatiquement sont souvent mises à mal, et qui ne connaissent que peu de visibilité pour ce qu'elles sont, c'est-à-dire, comme expression dans un espace public oppositionnel, un agir venu de la démocratie sauvage, une démocratie qui agit contre l'État.

Il faut également insister sur l'aspect fondamental qui permet cette réception de l'espace public oppositionnel quand les couches populaires et moyennes se rencontrent et partagent des intérêts communs, alors la perception dans la sphère classique de réception est modifiée.

83 Il y a là comme un levier politique qui ne peut être ignoré : les classes moyennes sont en quelque sorte la strate de la société sur laquelle la démocratie représentative trouve sa légitimité, il devient alors impossible de totalement rejeter les phénomènes et manifestations auxquels elles participent. L'exemple argentin permet de le penser et de le souligner. Car, le contre l'État, qui est en jeu dans les manifestations et les soulèvements en Argentine, est ainsi suspendu aux lèvres de ces gens qui appartiennent bien à l'espace public oppositionnel, mais qui ne peuvent être réprimé avec la même violence aux vues de leur place dans la structure sociale et du fait qu'ils sont à l'origine du système politique démocratique moderne.

L'espace public oppositionnel est particulièrement intéressant en Argentine parce qu'il réussit à associer dans des mouvements communs la société et la démocratie participative 
avec un demos sauvage. Tous sont concernés et les manifestations, et autres expériences de rue, voient des actions de concert comme l'évoque Abensour (Abensour, p.109).

Il y a là une rencontre de l'hétérogénéité sociale, qui consolide l'espace public oppositionnel. Avec une société où il y a $50 \%$ de gens pauvres, les implications sont plus directes. Quoiqu'il en soit, la « classe moyenne » est mobilisée, attentive et les traditions progressives qui existent à la manière d'un courant souterrain dans l'histoire du pays, ont pu resurgir au grand jour en 1983 ou 2001. Il y a eu aussi une incroyable association de la créativité et de l'inventivité des acteurs. La richesse de la lutte politique en Argentine se tient là, dans ce nœud entre oppression économique et oppression politique, entre injustice morale et injustice sociale qui ne laisse pas les citoyens en lutte indifférents.

Par ailleurs, l'histoire particulière de l'Argentine est aussi un barrage à l'usage inconsidéré de la violence, car il viendrait mettre en danger la forme même de la démocratie, en faisant flotter sur la répression le spectre de la dictature - qui est profondément rejeté par la société actuelle.

Néanmoins, je pense qu'en réalité, il y a dans les démocraties modernes un réel rejet des couches populaires (les classes dangereuses) et une violence agissante qui n'est pas assez clairement saisie, puisque le mouvement de l'espace public traditionnel-bourgeois est non pas de permettre le saisissement de la réalité, mais de créer un double de cette réalité occultant les enjeux politiques (et aussi une partie des acteurs politiques - les « exclus »).

L'espace public oppositionnel est donc profondément relié à cette catégorie à la fois plébéienne et marquée par sa profonde altérité : Non seulement porteuse d'une culture autre, mais aussi manifeste par des signes extérieurs qui la cantonnent à une identification d'étranger ou de citoyen de deuxième catégorie. Il est manifeste qu'un des enjeux de cette conceptualisation est pour nous de pouvoir rendre ce qui appartient à cette partie de la société : sa capacité à agir, sa capacité politique. Il s'agit de saisir l'enjeu dans nos sociétés des raisons de nier et d'occulter les formes particulières de la politique qui existent et s'expriment chez ces acteurs sociaux à part entière.

Il faut aussi s'interroger pourquoi Montréal-Nord, pourquoi cette partie du Canada, qui connaît des pratiques progressistes et qui pourtant a aussi des difficultés particulières avec les jeunes issus de l'immigration.

90 Par exemple, le fait que le Québec demande de signer une Charte à ses immigrants, et qu'il veuille ainsi s'assurer de l'adhésion des étrangers ou nouveaux arrivants à «ses valeurs » est sans doute un des points qui expriment la relation ambivalente entretenue avec cette part de sa population venant d'ailleurs. La peur de disparaitre en tant que «nation » est aussi agissante dans cette relation :par exemple dans ce souci de perdre la langue française et ainsi une part de son identité est aussi associé à cette arrivée de migrants qui ont sans doute un meilleur -croît-on- usage de l'anglais.

91 La loi 101 est une de ces trouvailles pour endiguer cette disparition, mais cette relation inquiète à la langue entraîne sans doute des relations ambivalentes à l'arrivant. Les révoltes de Montréal-Nord ne sont pas le fruit du nouveau banditisme québécois, vu et représenté comme "haïtien", mais bien plutôt la manifestation d'un rejet d'une stigmatisation négative diffuse et raciste que la société québécoise à du mal à reconnaître en son propre sein, puisqu'elle est ouvertement condamné ${ }^{15}$.

92 L'inversion opérée par l'État et l'espace public bourgeois est assez proche de celui de France. En effet, dans des sociétés qui connaissent à différentes échelles une précarisation des conditions de vie, généralisées, la réponse est de détourner la peur de l'opinion 
publique de ce qui menace réellement les individus : des conditions de vie de plus en plus précarisées, un univers du travail et un marché de la production qui connaît une rupture historique avec l'essor du capital financier. À cette peur légitime, l'État et l'espace public bourgeois font une sorte de tour de passe-passe, et agite la peur de l'autre, de l'étranger et du desconocido. Le danger est mis au mauvais endroit, à un endroit qui historiquement a toujours excellemment bien fonctionné : stigmatiser l'Autre comme étant un danger.

Ainsi, en France, le « musulman », par exemple, est devenu celui par qui la société laïque peut être " détruite ». Car, il possède « une religion active, qui cherche à prendre la place de l'autre, à dominer ", il ne "respecte pas les fondements laïques de nos sociétés » et il est en capacité dans les fantasmes de l'opinion publique de défaire notre société si bien portante jusqu'à son arrivée. Cette perception cache donc ce dont il faut vraiment avoir peur - les conditions de vie des gens - et nous fait oublier ce contre quoi il s'agit de lutter. À contraposto il est véritablement impressionnant de remarquer, en France, combien les personnes issues de l'immigration ou migrantes sont en fait à la source de luttes politiques actuellement. Les actions des sans-papiers irriguent par exemple la question des conditions de travail et nourrissent une lutte plus vaste pour qui touche à la condition $\mathrm{du}$ monde du travail. Les jeunes des banlieues, pris dans un rapport où ils subissent la violence de l'État, sont les premiers à lutter contre les abus du pouvoir et à être dans une pratique de résistance et de liberté par les pratiques. Il y a fort à parier que les choses politiques- ne vont pas s'arrêter là, et qu'il ne s'agisse que d'un début.

95 Le durcissement de l'État, vers une forme qui prend de plus en plus de pouvoir sur les individus et les libertés, ne cachant plus son caractère autoritaire, en France est aussi le fruit de conditions et d'un contexte général extrêmement favorables avec la période des administrations Bush, après les attentats du 11 septembre.

Il ne faut pas ignorer cette direction généralisée et l'impact de la locomotive du «monde libre » sur le reste des pays, notamment de l'Europe. Il ne faut pas non plus exclure que le droit que les USA se sont donné de ne pas respecter les droits des prisonniers dans leur lutte contre le terrorisme, a eu des effets considérables dans le monde occidental. Non seulement, sur les directions politiques des États, mais aussi pour ce qui caractérise la forme étatique -avec une période d'inflation des pouvoirs allant jusqu'à l'abus. L'élection de Barak Obama est sans doute la promesse d'un renversement de tendance et peut apporter des changements au sujet de l'inflation des pouvoirs étatiques pour certains pays comme la France ${ }^{16}$.

97 La question actuelle et la limite de cette « désinflation étatique » repose sur l'absence de réaction de la société et de l'opinion publique. Si l'État trouve l'apathie ou la complaisance des foules, alors il est fort à parier que cette situation, qui est aussi le signe d'une crise de la démocratie, ne sera pas arrêtée par les institutions elles-mêmes.

La première qualité de ces manifestations est celle d'exprimer une révolte contre une situation sociale où l'injustice et l'iniquité dominent. Il s'agit de rendre compte que ces manifestations sont autant d'expression des souhaits et des intérêts d'une partie de la société mise à mal et écarter par l'ensemble de la société et l'ordre social. Ces manifestations sont donc l'expression d'un contre l'État, dans le sens où elles sont là pour exprimer leurs désaccords avec l'État, leur opposition à la forme actuelle de l'État durcissement dans le cas français et canadien- et dépolitisation et corruption de la forme étatique dans le cas argentin. 

luttent contre la dynamique de l'État lorsqu'elle va vers des aspects autoritaires, et elles
expriment aussi un désir de liberté et de démocratie qui est constitutif à ce qui a entraîné
l'apparition de cette forme étatique, et qui doit toujours rester vivace dans l'État.

\section{Fragments de l'expérience}

, dernier point d'analyse, qui est rendu possible grâce à Negt, c'est la pensée critique de la fragmentation de l'expérience. Ainsi, je pense qu'il faut mettre en relation les révoltes des banlieues et la lutte contre le $\mathrm{CPE}$, car il y a eu là une rencontre des sphères et des luttes qui ont pu se combiner et se croiser pour permettre aux jeunes de remporter une victoire - au moins symbolique et politique si ce n'est économique et sociale - sur un gouvernement qui les méprise. Il y a donc des zones perméables de rencontre démocratique. Des espaces potentiels, où, pour les plus exclus, il est possible de faire l'expérience de la politique et du dépassement. En espérant que cela participe à leur donner des expériences qui leur permettent de saisir les formes "convenues" ou « audibles» de la lutte politique, car il est à souligner que les acquis sociaux sont le fruit des résistances, des luttes et de la désobéissance au pouvoir et que rien n'est jamais venu de la docilité et la soumission.

Pour autant, les problèmes de violence, de sa dynamique et de ses effets fortement archaïques, ne doivent pas être ignorées, dans une représentation_angélique des exclus. La violence doit aussi se penser en termes d'identification à l'agresseur. Cette projection est aussi fortement privilégiée par des médias, qui jouent sur un renversement la réalité de la domination et de l'exclusion, la violence subie étant niée au profit d'une représentation archaïque et négative d'un exclu devenu le premier ennemi public. La question du terrorisme et de la situation mondiale ayant renforcée cette perception propice à enflammer le terreau fertile du racisme ordinaire français.

102 L'espace public oppositionnel est le territoire commun partagé et différenciée de formes différentes, opposées, contradictoires et parfois complémentaire de l'expression politique des subjectivités rebelles ${ }^{17}$.

103 Nous allons maintenant nous interroger sur cet aspect de la violence qui laisse la démocratie sauvage aux prises avec ses formes archaïques.

\section{Le point aveugle : l'usage de la violence et les limites de l'expression populaire de la politique}

104 Il faut cependant considérer l'aspect profondément néfaste -et non pas négatif- qui est aussi en action dans les expressions des couches populaires : c'est l'usage de la violence. En effet, les pratiques politiques ne sont pas exemptes de pratiques aliénées, et c'est là que réside un problème, car l'usage de la violence et les pratiques archaïques coexistent dans les couches populaires ${ }^{18}$. Ainsi, les formes peuvent toujours se retourner et se renverser en leur contraire. Machisme, sexisme, antisémitisme, pour les jeunes de banlieues.

La paupérisation et la désespérance dans laquelle se trouvent les couches populaires est de plus en plus grande. Je tiens à signaler la relation entre l'abandon dans lequel se trouvent les populations et l'essor de fait-divers de barbarie. Ainsi le jeune Ilan a été 
séquestré et torturé jusqu'à la mort, parce que juif, il devait " posséder de l'argent ", ce fait-divers véhicule bien la montée de la haine de l'autre, et notamment elle s'exprime dans l'antisémitisme, dans les zones urbaines où les gens sont abandonnés à leur désespoir. Il faut aussi indiquer que l'un des meurtriers d'Ilan a été, enfant, victime de sévices de la part de son beau-père et que les enseignants de son collège ont interpellé les services sociaux, qui leur ont répondu que, aux vues, de l'âge du jeune garçon, 12-13 ans, il était trop tard pour intervenir.

La haine peut aussi diriger les actions, pas seulement l'indignation ou la colère dont parle Proust qui théorise une pensée de la résistance axée sur les sentiments négatifs, mais médium conduisant à la résistance, au désir du « que cela cesse » et au souhait de justice et de liberté. Dans le même temps, ces sentiments négatifs peuvent aussi conduire à des conduites archaïques et - ou haineuses et créent une dynamique conduisant à la violence.

L'impact néfaste de celle-ci est double : incorporer des discours et attitudes -pratiques de haines déplacées et venues des médias et autres groupes sociaux que l'on subit et les retournés vers une altérité -devenant bouc émissaire. Dans ce moment-là, alors, il y a ce mouvement néfaste qui peut accaparer l'action politique populaire et se retourner contre elle, c'est-à-dire, qu'elle va alors cesser de porter un projet de dépassement et se retourner en son contraire ${ }^{19}$. En effet, rien ne garantie qu'une révolte qui est en soi une brèche dans l'ordre établi ne se renverse pas en son contraire. C'est ce qui est toujours difficile à saisir, et qui existe aussi par exemple dans la problématique des années 70 quand pour changer le monde il est théorisé et mis en place qu'il faut passer par l'usage de la violence contre l'état et quand cette violence devient une violence contre les individus.

Ce même usage de la violence est ainsi ce qui permet et redonne de la légitimité à l'État pour faire usage de son monopole -reconnu et légitime- de violence. Ainsi, aux situations de violence contre l'État par le biais des individus qui le représentent ou le «Grand Capital ", alors l'État trouve dans des parts larges de la population un certain assentiment à réprimer la violence. Le problème est encore actuel, avec les procès des anciens groupes armés italiens en Europe, et il connaît aussi des points de visibilités dans les théorisations, par exemple, sur l'Argentine, quand durant les années 80, certains courants d'intellectuels renvoient dos à dos l'État et les groupes armés.

L'usage de la violence trouve ainsi une double compréhension, il est toléré jusqu'à un certain point et à d'autres moments, il est rejeté et dans ce moment-là, l'État connaît alors un soutien de la part de la société. La violence peut donc dans ces temps-là être instrumentalisée par l'État, et trouvée ou non la légitimité populaire.

110 Ici, sans doute, il y a aussi un lien possible à faire avec la question du non verbal et la particularité aussi d'être ou de venir de population migrante qui peut aussi renforcer une problématique d'atonie ${ }^{20}$. Cette difficulté d'exprimer une pensée politique est ainsi traversée entre la problématique du non entendu propre à l'espace public oppositionnel populaire. Elle est traversée par la question de la langue aussi. Enfin, par le lien avec des situations de détresse social et psychique très grandes ou trop grandes. De l'insupportable des conditions de vie. 


\section{La ville, lieu de vie symptomatique}

111 Je pense que le croisement du travail de Negt avec celui de Mike Davis serait une manière dialectique d'accompagner cette prise de vue des « révoltes urbaines ». En effet, dans son livre, Au-delà de Blade Runner, Davis s'interroge sur la fragilité des conditions de vie, à l'intérieur des centres urbains ${ }^{21}$. Il constate le renversement entre une politique qui investit de plus en plus dans le domaine de l'insécurité ou de la sécurité et le choix d'un renoncement dans les investissements pour une politique sociale (Davis, pp. 92-93).

112 Ainsi, cet abandon politique du mieux vivre est à l'origine d'une situation économique et sociale inextricable qui conduit à la violence. Impossible de ne pas faire le lien avec la déréalisation de l'expérience urbaine faite à Los Angeles et de cette dimension sécurité. Le fantasme de la peur est la source sur laquelle la politique de répression sociale s'appuie. Un monde imaginé où l'altérité est un fléau. Le délitement de la vie urbaine rencontre donc les émeutes-révoltes de 1992, suite à l'agression de Rodney King (Davis, p.28).

113 Il y a ainsi la jonction entre cette politique de répression et sécuritaire et la mise en place de ghettos. Donc, il y existe une scission sociale entre les couches de la société et un retranchement de ce qu'on appelle «classes moyennes». Deuxièmement, une déréalisation de la ville où le réel s'évapore au profit d'une simulation accrue -le fairesemblant social-proche de l'analyse de la double réalité de Negt (Negt, pp.155-177).

Le contrôle social est ainsi le nouveau ciment sur lequel se construit la cohésion sociale, la nouvelle place donnée à un ennemi intérieur source de tous nos maux et une déréalisation de l'expérience urbaine et de l'expérience en général qui permet de faire interagir les individus sur des modes régressifs et archaïques. Il y a là une grande proximité avec le travail d'un Henri Lefebvre ${ }^{22}$ qui dénonçait déjà la déshumanisation de l'homme par l'urbain alors même que la ville a été le lieu de la révolution, comme Walter Benjamin l'avait souligné dans Le livre des passages ${ }^{23}$.

115 Pourquoi ces révoltes à Montréal Nord ? Il semble, il est clair, qu'ils sont l'expression d'un dysfonctionnement et le signal des effets de cette dynamique politique archaïque qui avance également dans le discours de certains partis au Canada. Cette société qui a été en quelque sorte jusqu'ici assez épargnée et rattrapée par les nouvelles formes de la démocratie contemporaine, marquée par une nouvelle actualisation de la défiance vis-àvis de l'altérité.

La ville américaine (USA) est brillamment saisie par Davis. Il nous montre bien comment s'établit un lien entre d'un côté le pouvoir économique et l'organisation de la cité. Ainsi, les centre-villes sont-ils laissés à l'abandon et aux pauvres, au profit des banlieues pour les classes moyennes et favorisées. Il dévoile la correspondance, entre la désespérance sociale, l'apparition du ghetto et de villes-fantômes, et les décisions politicoéconomiques. Ainsi, le désinvestissement public concernant tant l'éducation que le cadre urbain des couches populaires, s'accompagne de choix et de décisions: Préférer l'investissement dans les forces de police, la répression, les prisons est nettement plus profitable aux ré-élections. La criminalisation des couches populaires, la haine de l'Autre - identifié comme noir et délinquant- toutes ces attitudes actives d'une politique issue d'un rejet du multiculturalisme, conduisent la société moderne dans une impasse du vivre ensemble. C'est la haine et l'usage de la peur de l'autre. L'inflation des pratiques de 
contrôle et la mise en place de la surveillance maintient et participe à ce déploiement anxiogène face à l'autre. La phobie sociale s'est irrémédiablement installée en Californie, vidant la ville de son rôle de lieu d'échange.

117 La construction en France est différente, mais il est à noter que la politique qui pointe derrière les décisions prises est la même. Même projet social et urbain marqué par la peur et la haine de l'autre, ici identifié aux « arabes et noirs et délinquants ». Même attitude de renforcement du sentiment de peur et le besoin de contrôle face à l'expérience d'altérité. Les sociétés modernes ont ainsi cette marque de ne pas savoir comment réceptionner l'altérité et la différence. Le discours anxiogène face aux migrants ou français d'origine étrangère est pour ainsi dire le même. Cette question du multiculturalisme est au cœur de cette crise du vivre ensemble.

118 Ainsi, non seulement les besoins de contrôle et de surveillance croissent dans les centrevilles -lieu où vivent les couches sociales plus favorisées- mais l'abandon des zones périphériques où sont installées les couches populaires est aussi très forte. Il $\mathrm{y}$ a au contraire une mise à l'écart dans des zones éloignées des centre-villes des jeunes des couches populaires. La ville s'érige protégée des foules de «barbares » et les populations sont abandonnées pour ainsi dire à leur sort dans des zones reculées. Les biens communs sont détériorés, souvent ils ne sont pas remis en service, les jeunes sont sans cesse contrôlés et souvent humiliés par des forces de l'ordre qui ont des attitudes de plus en plus violentes à leur égard. La peur que véhicule la police chez les migrants est si forte que certains se jettent par les fenêtres et meurent avant qu'elle ait pénétré dans leurs domiciles. La criminalisation des populations populaires, par le durcissement des règles pour obtenir des papiers de résident, l'usage de la législation pour retirer à certains des droits de résidence obtenus depuis des années, le traitement des enfants et des mineurs ayant des problèmes de statuts.

119 Cette politique française trouve une redondance dans les choix de l'Europe. Une loi européenne permet désormais de renvoyer un mineur dans son pays d'origine même si celui-ci n'y a aucun tuteur. Tous ces éléments participent à faire vivre les couches populaires dans la terreur. À laquelle s'ajoute la désespérance sociale de ne pas trouver un travail, de vivre de perpétuelles discriminations à l'embauche, de subir les contrôles policiers et d'être considéré comme une population potentiellement dangereuse - les musulmans ayant subi la stigmatisation d'être identifié comme de possibles terroristes par exemple. Ainsi, certains ont été congédiés de leur travail aux aéroports internationaux à cause de leur origine, pressentie comme une menace possible.

Il faut aussi souligner qu'au contraire, là où les couches populaires vivent dans la cité en ayant un échange avec les autres populations, il n'y a pas eu ou peu de révolte, ainsi à Paris dans le quartier de la Goutte d'Or ou encore à Marseille. Ainsi, le fait de vivre ensemble change aussi la relation à la société. Mais à Paris, on assiste à une restructuration de la ville où les populations des quartiers populaires sont chassées en douceur de la cité. La ville Paris -concentration des populations plus favorisées et des touristes de passages- tend à écarter ces populations populaires, mais elle connaît aussi désormais une réapparition des problèmes des couches populaires, malgré toutes ses stratégies d'isolement urbain qui accompagne l'isolement social. La gare du Nord est ainsi devenu le point par lequel les classes populaires s'engouffrent dans la cité, la porte d'accès. Depuis, 2005, un certain nombre de mini-actes de révoltes et aussi de vandalisme ont eu lieu dans la gare. Comme dans les films de science fiction, la porte d'entrée qu'est la gare du Nord, connectant ainsi les couches populaires à leur lieu de travail, est ainsi 
devenu le point faible de cette construction de l'espace qui est aussi une mise à l'écart des populations. Désormais, il est devenu normal de voir des militaires en mitraillette se promener dans ses couloirs, des forces de polices et des forces de sécurités propres aux entreprises de transport RATP/SNCF. Il faut ainsi ajouter que les échauffourées avec les forces de l'ordre ont eu lieu à la suite d'incidents, où ces forces de répressions commettaient des abus de traitements sur des passagers - identifiés comme migrant ou d'origine étrangère. Le saccage qui s'ensuit -où des jeunes cassent les vitrines des petits commerces pour se saisir de chaussures de marques ou de cellulaires- s'inscrit dans un double mouvement. D'abord, ces vols permettent de discréditer ces jeunes - car il est scandaleux de voler des objets vécus comme du luxe- et deuxièmement de signaler une part de ce à quoi ils aspirent: Avoir une place dans cette société qui est une société de consommation.

La ville dans de nombreux endroits de l'Amérique latine connaît aussi un clivage richepauvre. Ainsi, les bidonvilles se construisent en général à la périphérie de la cité. Dans un " en-dehors ". Il y a là encore une formation en occultation. Les habitats des pauvres sont tenus éloignés et ils sont ostracisés par la structure spatiale. La cité de Buenos Aires se construit dans une forme mixte entre des banlieues résidentielles et riches, sans pour autant abandonner complètement la ville aux couches populaires. Il y a donc un modèle différent, mais avec le même propos de mise à l'écart des individus en fonction de leur situation sociale. D'ailleurs, c'est de cette séparation sociale du vivre ensemble que peuvent s'appuyer les formes anxiogènes de la peur de l'Autre et du développement des formes de la « sécurité » et du contrôle.

Le quartier de Montréal-Nord est bien différent, les migrants et population d'origine étrangère qui y vivent n'ont pas encore été relégué loin de la cité. La société québécoise et canadienne a encore en elle les traces d'une société encore relativement protégée ${ }^{24}$. Cependant, les problèmes de disparitions de travail peu ou pas qualifié, la tendance générale à asseoir la politique sur la peur de l'autre, à agiter le spectre de la disparition de l'identité et la menace perçue comme venant de l'altérité et du multiculturalisme, tous ces éléments ne sont pas des signes rassurants, quant à la place donnée à cet Autre, celui qui est différent.

Il serait intéressant à poursuivre cette piste de la déshumanisation du cadre de vie initiée par Henri Lefebvre avec son livre Le droit à la ville, qui s'inscrit aussi dans la problématique réalisée par Davis, et en la complexifiant avec une réflexion sur ce que furent les villes -espaces des révolutions- perçu et analysé par Benjamin dans son Livre des passages ${ }^{25}$. Il y a sans doute ici une réflexion à conduire sur le lieu de vie et son adéquation avec les directions politiques de gouvernance.

Il faut aussi remarquer cette intrusion, commune aux USA et en France notamment, sur l'intervention de plus en plus systématique des forces de répressions dans les choix architecturaux des espaces communs et des lieux de vie pour les populations populaires. Ainsi, est paru un article dans Le Monde en 2008 faisant état des directives urbanistiques données par les forces de police afin de pouvoir intervenir aisément en cas d'émeute ou de crise. Davis nous montre la même chose concernant la Californie (Davis, p. 56-77).

Il faut ainsi penser ce double envahissement de l'espace commun et des lieux de vie par d'un côté la marchandisation qui occupe et envahie l'espace urbain, ne laissant plus un seul espace vide pour la contemplation, et sa conjugaison avec un espace pensé pour permettre la répression. C'est comme si l'individu ne pouvait échapper à ce carcan social. 
Nous sommes bien loin des promenades d'un Baudelaire ou de Benjamin, nous sommes aux prises constantes avec les messages aliénant qui parcourent l'espace urbain.

Pour autant, nous sommes actuellement dans une situation de crise - économique et politique mondiale - un nouveau Président aux USA a été élu, il est le signe d'un possible tournant. Barak Obama porte en lui de telles promesses que je pense que d'une certaine manière, l'analyse est aussi suspendue à ces lèvres, car il y a de réelles interférences entre ce qui se joue là-bas et ce qui va se jouer pour nous tous. Les États démocratiques contemporains parviendront-ils à repenser le vivre-ensemble-s sur d'autres bases que la peur et la haine ? Est-ce que ce nouveau président des USA sera en capacité d'infléchir ce virage politique en construisant un chemin différent auquel nous pourrons tous nous référer? L'Europe va-t-il transformer sa position phobique et répondre différemment aux crises actuelles? Il est trop tôt pour le savoir, mais il est nécessaire de rappeler que cette dynamique étatique des démocraties contemporaines porte, en elles, tous les éléments propices à des situations de violence et de rejet d'autant plus exacerbées qu'une crise économique se déploie au même instant.

\section{NOTES}

1. Oskar Negt, L'espace public oppositionnel, Payot, 2007, textes choisis, traduit et introduit par Alexander Neumann.

2. Terme repris à Alexander Neumann in « Oskar Negt et le courant chaud de la Théorie critique : Espace public oppositionnel, subjectivité rebelle, travail vivant », op. cit.

3. Il faut souligner la multiplication de fait-divers en France, concernant la fuite de personnes devant une interpellation policière, dont certaines ont entraîné la mort.

4. Hourya Bentouhami, "La justice sociale face à la question en banlieue », in L'autre campagne, 2006-2007, voir w. lautrecampagne.org..

5. Une table ronde a été, le jeudi 30 Octobre 2008, organisée par la Chaire de recherche sur l'immigration, l'ethnicité et la citoyenneté. Sous le thème de « Montréal-Nord, du conflit urbain à l'humanisation du quartier? Une crise annoncée».

6. P. Clastres, La société contre l'État, éditions de Minuit, chap. I, 1974.

7. Hourya Bentouhami "La justice sociale face à la question en banlieue ", in L'autre campagne, 2006-2007, voir www.lautrecampagne.org.

8. Eustache Kouvélakis, Luttes sociales et cycles politiques 1986-2006, Paris, Textuel, 2007. Yves Sintomer, préface du livre de Will Kymlicka, La citoyenneté multiculturelle, une théorie libérale du droit des minorités, 2001. Alain Bertho, Nous Autres, Nous-Mêmes, ethnographie politique du présent, éditions du Croquant, 2008. Didier Py, dir., Une révolte en toute logique. Des banlieues en colère, L'Archipel des pirates, 2005. Emmanuel Renault, Mépris social. Éthique et politique de la reconnaissance, Éditions du passant, 2000. Il ne s,agit pas d'une liste exhaustive, mais d'une première indication de direction de lectures portant sur la question.

9. Françoise Proust, De la résistance, Les éditions du Cerf, 1997.

10. John Holloway, Épilogue, «Changer le monde sans prendre le pouvoir, » in Variations, éditions Parangon, Lyon.

11. Miguel Abensour : La démocratie contre l'Etat. Marx et le moment machiavélien, le Félin, 2004. 
12. Il est intéressant de noter que cette idée de démocratie sauvage souvent mise en relation avec Lefort est en fait aussi une transformation de la pensée de Clastres ou disons sa continuation, car Clastres donne une interprétation de la politique où se préoccupant des sociétés amérindiennes, et plus précisément guarani, il réalise en fait une conceptualisation $\mathrm{d}$ une lutte pour le rejet de l'apparition du rapport de coercition par la société, ce qui vaut à son ouvrage le titre de la société contre l'État, que, Abensour poursuit dans son ouvrage La démocratie contre l'État.

13. Claude Lévi-Strauss, La pensée sauvage, Agora Pocket.

14. Alessi Dell'Umbria, C'est de la racaille ? Eh, bien j'en suis, L'Échappée, 2006.

15. Cette analyse était aussi faite lors de la table ronde de l'UQAM.

16. La fermeture du centre de rétention de Guantanamo est d'ailleurs une de ses premières décisions en tant que futur président des USA. Elle me semble refléter ce changement de direction politique qui sera, dans son effectivité, sans doute le signe d'un arrêt de cette dynamique néfaste des formes démocratiques contemporaines.

17. Francis Dupuis-Déri, dir., Québec en mouvements, Futur proche, Lux, 2008.

18. John Holloway, Changer le monde sans prendre le pouvoir, Lux éditions, 2008.

19. Cette idée est reprise du travail de la Théorie critique et d'Adorno en particulier.

20. Abdelmalek Sayad, La double absence, Des illusions de l'émigré aux souffrances de l'immigré, Seuil, 1998.

21. Mike Davis, Au-delà de Blade Runner, Los Angeles et l'imagination du désastre, Allia, 2006.

22. Henri Lefebvre, Le droit à la ville, I, et II, espace et politique, Anthropos, 1968, et 1973.

23. Walter Benjamin, Paris capitale du XIXe siècle, Le livre des passages, Éditions du Cerf, 1986.

24. Joseph-Yvon, Thériault, Faire société, Sociétés civiles et espaces francophones, Agora, 2007.

25. Chacun de ces ouvrages a été cité précédemment.

\section{AUTEUR}

\section{LUCIA SAGRADINI}

Chercheuse en sociologie, Montréal 